\title{
Non-alcoholic fatty liver disease (NAFLD)
}

EASL-EASD-EASO

\section{Source}

. (2016). EASL-EASD-EASO Clinical Practice Guidelines for the management of non-

alcoholic fatty liver disease Journal of Hepatology, vol. 64 (6), 1388-1402.

doi:10.1016/j.jhep.2015.11.004

NAFLD is characterised by excessive hepatic fat accumulation, associated with insulin resistance (IR), and defined by the presence of steatosis in $>5 \%$ of hepatocytes according to histological analysis or by a proton density fat fraction (providing a rough estimation of the volume fraction of fatty material in the liver) $>5.6 \%$ assessed by proton magnetic resonance spectroscopy $\left({ }^{1} \mathrm{H}\right.$-MRS) or quantitative fat/water selective magnetic resonance imaging (MRI). NAFLD includes two pathologically distinct conditions with different prognoses: non-alcoholic fatty liver (NAFL) and non-alcoholic steatohepatitis (NASH); the latter covers a wide spectrum of disease severity, including fibrosis, cirrhosis and hepatocellular carcinoma (HCC). 